\title{
On the occurrence of colliding-wind binaries in $\mathrm{R} 136$
}

\author{
L. Norci ${ }^{1}$, J. Hartwell ${ }^{2}$ and E. J. A. Meurs ${ }^{2}$ \\ ${ }^{1}$ School of Physical Sciences, Dublin City University, Glasnevin, Dublin 9, IRL \\ email: lno@physics.dcu.ie \\ ${ }^{2}$ Dunsink Observatory, Dublin Institute for Advanced Studies, Castleknock, Dublin 15, IRL \\ email: ejam@dunsink.dias.ie
}

\begin{abstract}
Interpretative analyses of the X-ray emission from the giant starformation region R 136 have concluded that several colliding-wind binaries are likely to contribute to its Xray output. Using our dedicated high-energy stellar population synthesis programme, we try to reproduce the suggested number of colliding-wind binaries. It appears that only assuming a very high binary fraction for the cluster's stellar population we can reproduce the observed X-ray luminosity distribution, if also the two most luminous sources are in fact multiple sources.
\end{abstract}

Keywords. Stars:binaries, Stars:X-ray.

In a starburst region several components contribute to the X-ray emission. Single stars emit X-rays via shocked stellar winds or coronal emission. The evolved binary stars are stronger emitters, where the X-ray radiation is produced by an accretion process from an expanding normal star to a compact companion. Supernova remnants are also strong soft X-ray emitters, producing X-rays from shocked material. Stars emit X-rays at various levels in essentially every phase of their lives. Both the accretion process and $\mathrm{X}$-ray emission from the shocked material in a supernova explosion are instead short-lived occurrences.

The soft X-ray emission from stars has been extensively studied with the help of the data sets provided by the ROSAT Survey and is fairly well known. The binary stars present on the other hand a more difficult task. Whether the accretion process occurs or not in a certain binary system and with what modalities depends on a number of circumstances that are known with varying degrees of certainty.

Since the R 136 cluster is a very young cluster with an age between 1-2 Myr no supernova can yet have occurred and therefore interacting close binaries with a compact companion have not been produced either. The contribution of the colliding wind binaries has on the other hand to be taken into account.

We have simulated a realistic cluster for $\mathrm{R} 136$ composed of single stars and binaries. We have used for the evolution of the single star population the Geneva evolutionary tracks with metallicity $\mathrm{Z}=0.008$ and normal mass loss. We have assumed a Salpeter initial mass function for the single stars and the primaries of binary systems. A flat primary/secondary mass ratio distribution and a binary separation distribution which is flat in the logarithm have been adopted for this simulation. Spectral types are assigned following the temperature scale of Landolt-Börnstein.

The results presented here are produced assuming an instantaneous burst of star formation 2 Myr ago and a mass range $15-120 \mathrm{M}_{\odot}$. We have imposed, for the comparison with real data, that the distribution and number of stars above $50 \mathrm{M}_{\odot}$ must reproduce the observed mass distribution. The X-ray luminosity of colliding wind binaries is calculated with the formula of Portegies-Zwart et al. (2002). The observed X-ray luminosities 

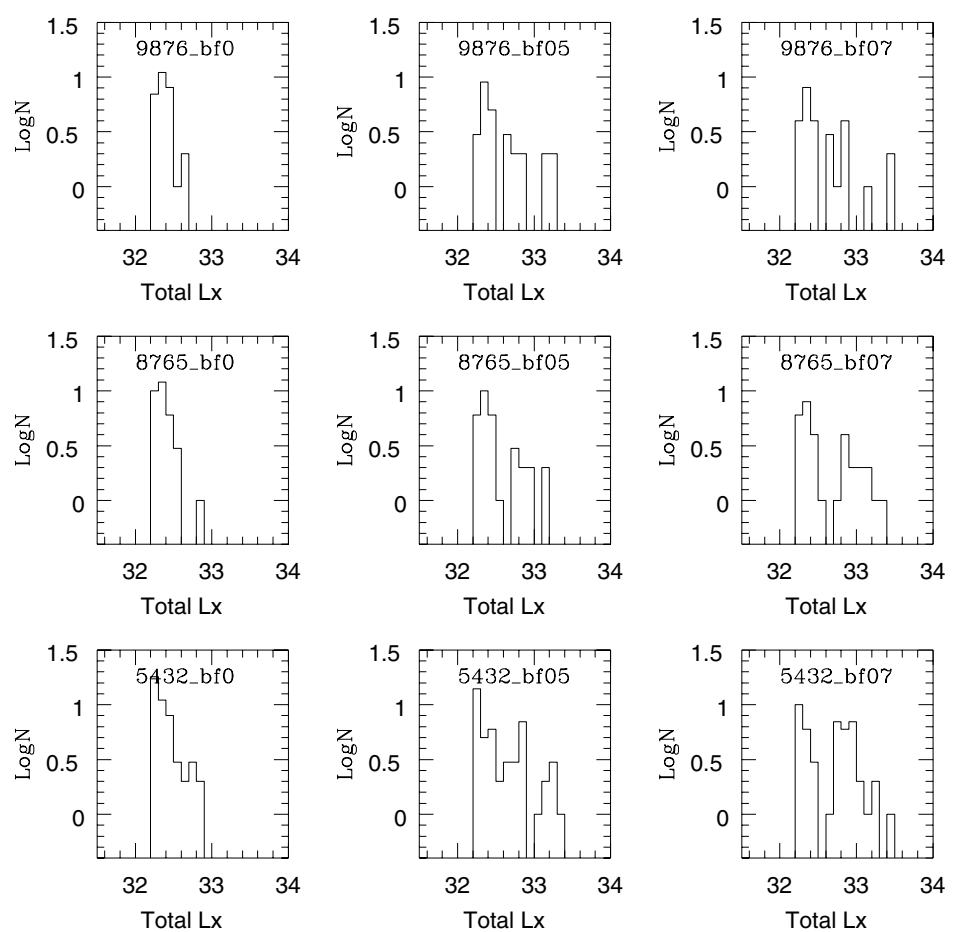

Figure 1: Simulation of the distribution of X-ray luminosities in the range of the observed Chandra sources; results are shown for three different initial mass distributions, including in the bottom row the simulation which we deem more representative of this cluster top masses.

From left to right the binary fractions increase as $0,0.5$ and 0.7 .

for the top masses in R 136 are taken from recent Chandra observations of the cluster region (Portegies-Zwart et al. 2002) and converted to the ROSAT range for comparison with our simulations. The mass distribution is taken from Vacca et al. (1996) and Chlebowski and Garmany (1991).

The results of our simulations are shown in Figure 1. These simulations show that a substantial number of binaries must be present if the top of the observed X-ray luminosity distribution is produced by colliding wind binaries.

In our simulations we assume that the most luminous X-ray sources in $\mathrm{R} 136$ are colliding wind binaries and that the two most luminous sources (above $10^{35} \mathrm{erg} \mathrm{s}^{-1}$ ) are in fact multiple sources, for which there is clear evidence for at least one of them. We conclude that the luminosity distribution of the remaining Chandra X-ray point sources is reproduced by our simulations only by assuming a very high binary fraction, around 1.0 , for the cluster stellar population.

\section{References}

Chlebowski, T. \& Garmany, C.D. 1991, ApJ 368, 241

Portegies Zwart, S.F., Pooley, D., \& Lewin, W.H.G. 2002, ApJ 574,762

Vacca, W.D., Garmany, C.D., \& Shull J.M. 1996, ApJ 460, 914 